Artigo / Article

\title{
Atividade da 6-fosfogliconato desidrogenase em deficientes de glicose-6-fosfato desidrogenase
}

\author{
Activity of 6-phosphogluconate dehydrogenase in glucose-6-phosphate dehydrogenase deficiency
}

Daniela B. Nicolielo ${ }^{1}$

Rosecler I.P. Ferreira ${ }^{2}$

Amauri A. Leite Le $^{3}$

\begin{abstract}
As enzimas G6PD e 6PGD são responsáveis pela geração do aporte de NADPH, necessário para a detoxificação dos agentes oxidantes produzidos pelo estresse oxidativo metabólico nos eritrócitos. Devido à alta prevalência de deficiência de G6PD na população mundial, principalmente de origem negróide africana, muitos estudos têm sido realizados na tentativa de conhecer melhor a atuação destas enzimas. O objetivo deste estudo foi avaliar a atividade enzimática da 6PGD, nos deficientes de G6PD, para verificar a existência de aumento da atividade desta enzima, correlacionando com um possível aumento do número de reticulócitos ou presença de alterações da série vermelha. A pesquisa em 2.657 indivíduos do sexo masculino resultou em 97 deficientes de G6PD, determinando uma prevalência de 3,65\% para a região de Bauru (SP), com atividade enzimática média de G6PD de 1,74 UI.g $H b^{-1} \cdot$ min $^{-1}$ a $37^{\circ} \mathrm{C}, 14,4 \%$ da atividade da G6PD normal. A atividade enzimática média da 6PGD foi de 9,5 UI.g $\mathrm{Hb}^{-1}$. $\mathrm{min}^{-1}$ a $37^{\circ} \mathrm{C}$, estando aumentada em 47,4\% dos deficientes de G6PD. Os resultados não confirmaram que a hipótese do aumento da atividade enzimática da 6PGD, em deficientes de G6PD, seja decorrente da presença de um número aumentado de reticulócitos na corrente circulatória, faixa etária ou alterações eritrocitométricas que denotem anemia. O mais provável é que a hemólise autolimitada, imposta pelos processos oxidativos, preserve os eritrócitos mais jovens, que possuem atividade enzimática mais elevada, uma vez que naturalmente ocorre diminuição da atividade destas enzimas com o envelhecimento celular. Rev. bras. hematol. hemoter. 2006;28(2):135-138.
\end{abstract}

Palavras-chave: Deficiência de glicose-6-fosfato desidrogenase; 6-fosfogliconato desidrogenase; reticulócitos; eritrócitos.

\section{Introdução}

O eritrócito é uma célula particular porque transporta oxigênio para os tecidos sem consumi-lo. A adenosina trifosfato (ATP), energia calórica, e a nicotinamida adenina dinucleotídeo reduzida (NADH) e nicotinamida adenina dinucleotídeo fosfato reduzida (NADPH), energia redutora, são obtidas a partir da oxidação anaeróbica da glicose, via das pentoses e ciclo da glutationa., ${ }^{1,2}$

A via das pentoses é responsável pela oxidação de cerca de $10 \%$ da glicose consumida pelo glóbulo verme- lho. A ação de duas enzimas seqüenciais, a glicose-6fosfato desidrogenase (G6PD) e a 6-fosfogliconato desidrogenase (6PGD) reduzem a coenzima nicotinamida adenina dinucleotídeo fosfato (NADP) à nicotinamida adenina dinucleotídeo fosfato reduzida (NADPH), sendo, praticamente, toda fonte geradora de NADPH. Ao manter a glutationa no estado reduzido (GSH), através da glutationa redutase, essa via desempenha importante papel na proteção do glóbulo vermelho frente aos processos oxidativos, que são responsáveis pela redução da vida média do eritrócito. ${ }^{2-4}$

${ }^{1}$ Centro de Ciências Biológicas e Profissões da Saúde da Universidade do Sagrado Coração - Bauru-SP. Mestre em Análises Clinicas pela FCF - Unesp. ${ }^{2}$ Núcleo de Atendimento à Comunidade: Hemonúcleo Regional de Araraquara da Faculdade de Ciências Farmacêuticas de Araraquara - Unesp-SP.

${ }^{3}$ Professor doutor do Departamento de Análises Clínicas da Faculdade de Ciências Farmacêuticas de Araraquara - Unesp-SP. 
A deficiência de G6PD é a mais freqüente enzimopatia conhecida, afetando aproximadamente 400 milhões de pessoas no mundo. Os eritrócitos deficientes de G6PD são incapazes de reduzir $\mathrm{NADP}^{+}$a NADPH em velocidade normal, apresentando assim um baixo potencial redutor, não conseguindo remover o peróxido de hidrogênio ou os dissulfetos mistos da hemoglobina formados após ingestão de certas drogas oxidantes e em alguns processos infecciosos e oxidativos. ${ }^{2}$ Ocorre hemólise autolimitada pela destruição dos eritrócitos senis, com conseqüente aumento de reticulócitos que apresentam maior quantidade da enzima. ${ }^{3}$

Este estudo tem por objetivo avaliar a atividade enzimática da 6PGD, nos deficientes de G6PD, para verificar se existe aumento da atividade desta enzima, correlacionando com possível elevação do número de reticulócitos ou presença de alterações da série vermelha.

\section{Casuística e Métodos}

Este estudo foi constituído por 2.657 amostras de sangue, coletadas por punção venosa a vácuo com o anticoagulante etileno diamino tetracético (EDTA), de indivíduos do sexo masculino que procuraram os serviços do Laboratório de Análises Clínicas da Universidade do Sagrado Coração, na cidade de Bauru. Foi realizada triagem populacional para deficiência de G6PD, através do teste de redução da meta-hemoglobina, técnica de Brewer, Tarlov e Alving, ${ }^{5}$ resultando em 97 indivíduos portadores de deficiência da enzima G6PD, que aceitaram participar da pesquisa, com parecer aprovado pelo Comitê de Ética em Pesquisa da Universidade do Sagrado Coração, conforme protocolo no $015 / 2003$.

Os participantes foram divididos em três grupos, de acordo com a faixa etária, possibilitando a comparação dos resultados obtidos no eritrograma com os diferentes valores de referência. O grupo 1 foi composto por 18 crianças com faixa etária entre 1 e 6 anos, o grupo 2 por 21 pessoas com idade entre 7 e 12 anos e o grupo 3 por 58 indivíduos com idade igual ou superior a 13 anos.

O eritrograma dos indivíduos deficientes de G6PD foi realizado em contador eletrônico de 22 parâmetros da marca Coulter, modelo Max'M e contagem manual de reticulócitos, utilizando o corante azul de cresil brilhante a $1 \%$.

A deficiência de G6PD dos grupos foi confirmada pela determinação da atividade enzimática, bem como a atividade enzimática da 6PGD. Ambas, segundo condições estabelecidas por Beutler, ${ }^{6}$ em espectrofotômetro Hitachi U-3000 em 340 nanômetros, no Laboratório de Hematologia do Centro de Referência Diagnóstica do Núcleo de Atendimento à Comunidade da Faculdade de Ciências Farmacêuticas da Unesp de Araraquara.

\section{Resultados}

A atividade enzimática média de G6PD e 6PGD encontrada para cada um dos grupos estudados, juntamente com a porcentagem da atividade calculada a partir dos valores de referência preconizados por Beutler, ${ }^{6}$ estão demonstradas na tabela 1. Em todos os grupos podemos observar aumento da atividade da 6PGD, com média de 9,50 UI.g $\mathrm{Hb}^{-1} \cdot \mathrm{min}^{-1}$ a $37^{\circ} \mathrm{C}$ ou $8,2 \%$, quando comparada com os valores de referência.

Tabela 1

Valores de atividade da G6PD e 6PGD e porcentagem de atividade em relação aos valores de referência ${ }^{6}$

\begin{tabular}{|c|c|c|c|c|}
\hline & \multicolumn{2}{|c|}{ Atividade G6PD } & \multicolumn{2}{|c|}{ Atividade 6PGD } \\
\hline & U.. ${ }^{*}$ & $\begin{array}{c}\% \text { da atividade } \\
\text { normal } \star \star\end{array}$ & U..$^{\star}$ & $\begin{array}{c}\% \text { da atividade } \\
\text { normal } \star \star \star\end{array}$ \\
\hline $\begin{array}{c}G 1 \\
N=18\end{array}$ & 1,80 & 14,9 & 9,64 & 109,8 \\
\hline $\begin{array}{c}G 2 \\
N=21\end{array}$ & 1,76 & 14,5 & 9,34 & 106,4 \\
\hline $\begin{array}{c}G 3 \\
N=58\end{array}$ & 1,66 & 13,7 & 9,52 & 108,4 \\
\hline Média & 1,74 & 14,4 & 9,50 & 108,2 \\
\hline
\end{tabular}

A tabela 2 demonstra o número de indivíduos com Hemoglobina (Hb) e Volume Corpuscular Médio (VCM) abaixo dos valores de referência recomendados e a Amplitude de Distribuição Eritrocitária (RDW), contagem de reticulócitos relativa ( 0,5 a $1,5 \%)$ e absoluta e a atividade da 6PGD acima dos valores, com o respectivo percentual de alteração, para cada parâmetro e grupo estudado.

Tabela 2

Valores eritrocitários de Hb, VCM, RDW, reticulócitos e atividade de 6PGD alterados, expressos quanto ao número e percentual

\begin{tabular}{ccccccc}
\hline & $\begin{array}{c}\mathrm{Hb} \\
\mathrm{g} / \mathrm{dL}\end{array}$ & $\begin{array}{c}\mathrm{VCM} \\
\mathrm{fL}\end{array}$ & $\begin{array}{c}\text { RDW } \\
\%\end{array}$ & $\begin{array}{c}\text { Ret. } \\
\%\end{array}$ & $\begin{array}{c}\text { Ret. } \\
\#\end{array}$ & $\begin{array}{c}6 \text { PGD } \\
\text { aumentada }\end{array}$ \\
\hline $\mathrm{G} 1$ & 4 & 5 & 5 & 2 & 0 & 9 \\
$\mathrm{n}=18$ & $22,2 \%$ & $27,8 \%$ & $27,8 \%$ & $11,1 \%$ & $0 \%$ & $50 \%$ \\
$\mathrm{G} 2$ & 8 & 1 & 0 & 3 & 0 & 11 \\
$\mathrm{n}=21$ & $38,1 \%$ & $4,8 \%$ & $0 \%$ & $14,3 \%$ & $0 \%$ & $52,4 \%$ \\
$\mathrm{G} 3$ & 5 & 1 & 5 & 8 & 3 & 26 \\
$\mathrm{n}=58$ & $8,6 \%$ & $1,7 \%$ & $8,6 \%$ & $13,8 \%$ & $5,2 \%$ & $44,8 \%$ \\
\hline Total & 17 & 7 & 10 & 13 & 3 & 46 \\
$\mathrm{n}=97$ & $17,5 \%$ & $7,2 \%$ & $10,3 \%$ & $13,4 \%$ & $3,1 \%$ & $47,4 \%$ \\
\hline
\end{tabular}


Dos 97 portadores de deficiência de G6PD, 47,4\% apresentaram aumento de atividade de 6PGD, 13,4\% no valor de reticulócitos relativo e apenas 3,1\% no absoluto. Também foi observada baixa incidência de alterações eritrocitométricas.

\section{Discussão}

Muitos estudos têm sido realizados na tentativa de conhecer melhor a atuação das enzimas G6PD e 6PGD, pela importância na geração do aporte de NADPH necessário para a detoxificação dos agentes oxidantes produzidos pelo estresse oxidativo metabólico e pela alta prevalência da deficiência de G6PD na população mundial, principalmente de origem negróide africana. Nossos estudos demonstraram que, em 2.657 indivíduos do sexo masculino, da região de Bauru (SP), submetidos à triagem para deficiência de G6PD, houve uma prevalência de 3,65\% deficientes.

Estudos anteriores realizados por Leite et al,7 em 3.106 doadores de sangue na região de Araraquara (SP), mostraram prevalência de 2,38\% deficientes de G6PD, enquanto Compri et al, ${ }^{8}$ pesquisando 4.621 amostras na comunidade de Bragança Paulista, encontraram 1,7\%. Outros estudos realizados em diferentes regiões do Brasil constataram prevalência em torno de $10 \%$ para os homens de ascendência africana e de $1 \%$ a $3 \%$ para os homens de descendência caucasiana. $^{9-15}$

Estudo realizado por Leite e Barretto ${ }^{16}$ evidenciou que indivíduos portadores de deficiência de G6PD A` apresentavam atividade de 6PGD 22\% maior que nos portadores da variante normal G6PD B. Porém foram utilizadas apenas oito amostras com a possibilidade deste aumento ter ocorrido devido a uma provável reticulocitose não investigada.

Os dados obtidos com os portadores de deficiência de G6PD mostraram que 47,4\% apresentaram aumento médio de 8,2\% na atividade de 6PGD. Apesar dos resultados apresentarem valores menores que os encontrados anteriormente, demonstram claramente aumento na atividade enzimática da 6PGD.

Analisando os deficientes de G6PD em conjunto verificamos um aumento no número de reticulócitos em 13 $(13,4 \%)$ indivíduos no valor relativo e três $(3,1 \%)$ no absoluto. Nestes, a atividade da 6PGD apresentou elevação em cinco $(5,2 \%)$ e apenas em um (1\%), respectivamente.

Os resultados obtidos não confirmaram a hipótese do aumento da atividade enzimática da 6PGD, em deficientes de G6PD, ser decorrente da presença de um número aumentado de reticulócitos na corrente circulatória. ${ }^{3,7} \mathrm{O}$ mais provável é que a hemólise autolimitada, imposta pelos processos oxidativos, preserve os eritrócitos mais jovens que possuem atividade enzimática mais elevada, uma vez que, naturalmente, ocorre diminuição da atividade destas enzimas com o envelhecimento celular. Talvez isso possa ocorrer após episódios hemolíticos mais severos.
A possibilidade de deficientes de G6PD manifestarem considerável anemia hemolítica aguda na presença de fatores oxidantes foi o que objetivou correlacionar as alterações eritrocitométricas com o aumento da atividade da 6PGD.

Os resultados obtidos, quando somados os 3 grupos, mostraram que dos 17 (17,5\%) indivíduos que apresentaram hemoglobina abaixo dos valores de referência, a atividade de 6PGD aumentou em oito (8,2\%). Dos sete $(7,2 \%)$ com VCM abaixo dos valores de referência, a elevação esteve presente em cinco (5,2\%). Dos dez (10,3\%) com RDW acima dos valores de referência, estava presente em seis (6,2\%). Quando analisada conjuntamente a alteração nos três parâmetros encontramos dois indivíduos $(2,1 \%)$, sendo que apenas um (1\%) apresentou aumento da atividade da 6PGD. Apesar das alterações eritrocitárias encontradas nos grupos 1 e 2 serem mais freqüentes que no grupo 3, provavelmente isso ocorreu pela associação com outras formas de anemias, freqüentes em crianças e não avaliadas neste estudo.

Os resultados evidenciam a ocorrência de baixa incidência de alterações eritrocitométricas, não ocorrendo anemia ou alterações hematológicas importantes em indivíduos com deficiência de G6PD e aumento de atividade de 6PGD. Resultados obtidos por Sena e Ramalho ${ }^{17}$ também indicaram que a deficiência de G6PD não implica, necessariamente, em alterações clinicas e hematológicas severas, mesmo em constante contato com produtos oxidantes como sulfa, naftalina, etc.

\section{Conclusão}

A avaliação final dos dados produzidos por esse trabalho demonstra que o aumento da atividade da 6PGD, nos indivíduos deficientes em G6PD, não apresenta correlação com faixa etária, alterações hematológicas que denotem anemia ou presença de reticulocitose, que possam explicar o aumento da atividade da 6PGD, sendo mais provável que a hemólise autolimitada, imposta pelos processos oxidativos, preserve os eritrócitos mais jovens que possuem atividade enzimática mais elevada.

\footnotetext{
Abstract

The G6PD and 6PGD enzymes are responsible for the generation of NADPH supply necessary for the detoxification of the oxidant agents produced during the oxidative metabolic stress on erythrocytes. Due to the high prevalence of the deficiency of G6PD on world population, especially on Afro descents, many studies have been done trying to know better the actuation of these enzymes. The goal of this study was to evaluate the 6PGD enzymatic activity on a population with G6PD deficiency, to verify if there is an elevation of the activity of this enzyme, and try to correlate to a possible increase on the number of reticulocytes or the presence
} 
of alterations on red series. The research with 2657 male individuals detected 97 deficient for G6PD, which determined a $3.65 \%$ prevalence for the Bauru (SP) region, with mean enzymatic activity of $1.74 \mathrm{UI} . \mathrm{g} \mathrm{Hb^{-1 }}$. $\mathrm{min}^{-1}$ at $37^{\circ} \mathrm{C}, 14,4 \%$ of the normal G6PD activity. Mean 6PGD enzymatic activity was $9.5 \mathrm{UI}_{\mathrm{g} \mathrm{H}} \mathrm{Hb}^{-1}$. $\min ^{-1}$ at $37^{\circ} \mathrm{C}$, and was elevated in $47.4 \%$ of the G6PD deficient individuals. The result obtained did not confirm the hypothesis that the elevation of the 6PGD enzymatic activity, in G6PD deficient individuals, was due to the presence of an increase of reticulocytes in blood stream, age or erythrocytometric alterations that could denote anemia. The most plausible theory is that the auto-limited hemolysis, imposed by oxidative processes, preserves young erythrocytes that have an elevated enzymatic activity, as naturally these enzymes lose activity with cellular aging. Rev. bras. hematol. hemoter. 2006;28(2):135-138.

Key words: Glucose-6-phosphate dehydrogenase deficiency; 6phosphogluconato dehydrogenase; reticulocytes; red blood cells.

\section{Referências Bibliográficas}

1. Luzzatto L, Smith ECG. Inherited haemolytic anemias. In: Hoffbrand AV et al. Postgraduate Haematology. 4 ed. Oxford: Butterworth Heinemann, 1999:120-43.

2. Beutler E. Glucose-6-phosphate dehydrogenase deficiency and other red cell enzyme abnormalities. In: Beutler et al. Hematology. 6 a ed. New York: McGraw-Hill, 2001:527-39.

3. Lukens JN. Deficiência de glicose-6-fosfato desidrogenase e deficiências relacionadas que envolvem a via da pentose fosfato e metabolismo da glutationa. In: Lee RG, et al. Wintrobe Hematologia Clínica. São Paulo: Manole, 1998: 1101-13.

4. Mcmullin MF. The molecular basis of disorders of red cell enzymes. J Clin Path 1999; 52:241-4.

5. Brewer GJ, Tarlov RA, Alvin AS. Methaemoglobin Reduction Test. A new simple, in vitro Test for Identifying Primaquine-Sensitivity. Bull WHO 1960; 22:633-40.

6. Beutler E. Red Cell Metabolism: A Manual of Biochemical Methods. $3^{\text {a }}$ ed.: Grune \& Stratton, 1984, p188.

7. Leite ERM, Lessi JHC, Mascarin DB, et al. Pesquisa de deficientes em G6PD nos doadores de sangue da região de Araraquara. Bol Soc Bras Hematol Hemot 1996;18(supl):337.

8. Compri MB, Saad ST, Ramalho AS. Investigação renético-epidemiológica e molecular da deficiência de G6PD em uma comunidade brasileira. Cad Saúde Pública 2000; 16:335-42.

9. Azevêdo ES, Azevedo TFS. Glucose-6-phosphate dehydrogenase deficiency and neonatal jaundice in Bahia, Brazil. Ciência e Cultura 1974; 26:1.044-7.

10. Barreto OCO. Erythrocyte glucose-6-phosphate dehydrogenase deficiency in São Paulo, Brazil. Rev Bras Pesq Med Biol 1970;3:61-5.

11. Garlipp CR, Ramalho AS. Aspectos clínicos e laboratoriais da deficiência de desidrogenase de 6-fosfato de glicose (G-6-PD) em recém-nascidos brasileiros. Rev Brasil Genet 1988;11:717-28.

12. Lewgoy F, Salzano FM. Dinâmica do gene que codifica a deficiência de G-6-PD na população de Porto Alegre. Ciência \& Cultura 1965; 17:152-7.

13. Marques J, Campos JO. Incidência da deficiência de glicose-6-fosfato desidrogenase em negros de Minas Gerais. Rev Ass Med Bras 1975; 21:111-2.

14. Ramalho AS, Beiguelman B. Deficiência de desidrogenase de 6-fosfato de glicose (G-6-PD) em doadores de sangue brasileiros. Folha Med 1976;73:281-2.
15. Saldanha PH, Nóbrega FC, Maia JCC. Distribution and heredity of erythrocyte G-6-PD activity and electrophoretic variants among different racial groups at São Paulo, Brazil. J Med Genet 1969;6: 48-54.

16. Leite AA, Barretto OCO. Atividade da 6-fosfogliconato desidrogenase eritrocitária em indivíduos deficientes de G-6-PD. Bol Soc Bras Hematol Hemot 1996; 18(supl):269.

17. Sena LLA, Ramalho AS. Clinical evolution of glucose-6-phosphate dehydrogenase deficiency in a Brazilian population. Braz J Genet 1985; 8:89-96.

Avaliação: Editor e dois revisores externos.

Conflito de interesse: não declarado

Recebido: 12/11/2005

Aceito após modificações: 30/01/2006 